\title{
Trapping of lower hybrid waves in elongated plasma depletions in the Earth's ionosphere
}

\author{
N. Borisov ${ }^{*, 1}$, F. Honary \\ Lancaster University, Lancaster, LA1 4WA, UK \\ Received 7 November 2007; accepted 23 November 2007 \\ Available online 5 December 2007 \\ Communicated by V.M. Agranovich
}

\begin{abstract}
Conditions for trapping of lower hybrid waves in plasma depletions in the Earth's ionosphere are discussed. It is shown that the trapping can take place for the frequencies not only below but also above the lower hybrid resonance frequency of the background plasma, which is important for the formation of the downshifted maximum of the secondary electromagnetic emission.
\end{abstract}

(C) 2007 Elsevier B.V. All rights reserved.

\section{Introduction}

Lower hybrid (LH) waves play an important role in space and laboratory plasmas due to their ability to interact efficiently with electrons propagating along the magnetic field lines and with ions in the transverse plane. Such waves are generated by different mechanisms (e.g., by electron or ion beams and also in processes of waves interaction and transformation). In the Earth's ionosphere LH waves can be excited at various heights. Localized bursts of LH waves (the so-called spikelets) were detected in the upper ionosphere by rockets and satellites in connection with the elongated plasma depletions [1-3]. It was argued that such spikelets could be trapped in plasma depletions. Possibility of LH waves trapping at the frequencies below the lower hybrid resonance (LHR) frequency of the ambient plasma has been investigated theoretically in the most simple form (cold plasma approximation, that is neglecting thermal corrections), see [4-6].

At lower altitudes (below the maximum of the F-layer) $\mathrm{LH}$ waves can also be generated artificially under the action of powerful HF radio waves [7]. The generated LH waves appear

\footnotetext{
* Corresponding author.

E-mail address: n.borisov@lancaster.ac.uk (N. Borisov).

1 On leave from the Institute of Terrestrial Magnetism, Ionosphere and Radio Waves Propagation (IZMIRN), Russia.
}

due to a sequence of nonlinear processes: excitation of the upper hybrid resonance (UHR) waves by linear conversion of an electromagnetic pump wave, formation of elongated plasma depletions (the so-called striations) in which the UHR waves are trapped, and the parametric decay of the UHR waves in striations resulting in the excitation of LH waves [8]. However, the mechanism of LH waves trapping in striations, similar to the trapping of LH waves in natural plasma depletions in the upper ionosphere [4-6] has not been reported in the literature. It is important that the trapping of LH waves could increase the efficiency of their excitation because the waves do not go far from the region of interaction (in the same manner as the trapping of the UHR waves in striations influence their intensity).

The aim of this Letter is to present a theory describing the trapping of LH waves in a warm plasma in elongated plasma depletions (either artificial or natural). This theory is based on the differential equation of the fourth order that takes into account thermal corrections. It will be shown that in such approach a new solution describing the trapping of LH waves with the frequencies above the local LHR frequency can be obtained. Such solution not provided in previous theoretical investigations, [46], is of prime importance because in ionospheric modification experiments LH waves with frequencies above the LHR frequency are supposed to be involved in the generation of secondary electromagnetic radiation-the stimulated electromagnetic emission (SEE). 


\section{Basic equations}

Our aim is to derive the linear differential equation describing the LH perturbations in plasma depletions taking into account thermal corrections. Ionospheric plasma is supposed to be weakly inhomogeneous. The magnetic field is assumed to be directed along the $z$-axis and the background inhomogeneity of plasma concentration is considered along the $x$-axis. As the LH perturbations are electrostatic ones, we introduce the electric potential in the form

$\Phi=\Phi_{0}(x) \exp \left[i\left(\omega t-k_{y} y-k_{z} z\right)\right]$.

Here potential $\Phi$ describes the electric field $\mathbf{E}=-\nabla \Phi, \omega$ is LH wave frequency, $k_{y}, k_{z}$ are the LH wave numbers along the $y$ - and the $z$-axes correspondingly.

For electrons we use the equation of motion

$i \omega \mathbf{v}_{\mathbf{e}}=\frac{e}{m} \nabla \Phi-\omega_{H e}\left[\mathbf{v}_{\mathbf{e}} \times \mathbf{H}\right]-\frac{T_{e}}{m N_{0}} \nabla n_{e}$

and the continuity equation

$i \omega n_{e}+\nabla N_{0} \mathbf{v}_{\mathbf{e}}=0$.

Here $\mathbf{v}_{\mathbf{e}}$ is the drift speed of electrons, $e, m$ are the electric charge and the mass of electron, $\mathbf{H}$ is the magnetic field, $\omega_{H e}$ is the Larmour frequency of electron, $T_{e}$ is the electron temperature, $N_{0}$ and $n_{e}$ are the background plasma density and the small perturbation of concentration of electrons caused by the LH oscillations.

Ions are non-magnetized since the LHR frequency $\omega_{\text {LHR }}$ is much larger than the Larmour frequency of ions $\omega_{H i}$. In such case, following the kinetic approach, the expression for the perturbation of the ion concentration $n_{i}$ that can be presented as

$n_{i}=-\frac{1}{\omega^{2}}\left[\frac{\partial}{\partial x}\left(\frac{e N_{0}}{M} \frac{\partial \Phi_{0}}{\partial x}\right)-\frac{e N_{0}}{M}\left(k_{y}^{2}+k_{z}^{2}\right) \Phi_{0}\right]$.

Here $M$ is the mass of ions. The link between the electric potential $\Phi_{0}$ and the perturbations $n_{i}$ and $n_{e}$ is given by the Poisson equation

$\nabla \mathbf{E}=4 \pi e\left(n_{i}-n_{e}\right)$.

Combining Eqs. (2)-(5) we are in a position to find a differential equation for the electric potential $\Phi_{0}$. In general case this equation is too complicated. Later on we shall concentrate on one particular case, assuming that the perturbations are strongly elongated in the direction of the magnetic field. Also we suppose that the frequency of perturbations is much smaller than the plasma frequency of ions $\omega \ll \omega_{P i}$. Hence the following approximate equation can be derived

$$
\begin{aligned}
& \rho_{e}^{2}\left(\frac{\partial^{2}}{\partial x^{2}}-k_{y}^{2}\right)\left(\frac{\partial}{\partial x} \frac{\omega_{P i}^{2}}{\omega^{2}} \frac{\partial}{\partial x}-\frac{\omega_{P i}^{2}}{\omega^{2}} k_{y}^{2}\right) \Phi_{0} \\
& +\frac{\partial}{\partial x}\left(\frac{\omega_{P e}^{2}}{\omega_{H e}^{2}}+1-\frac{\omega_{P i}^{2}}{\omega^{2}}\right) \frac{\partial}{\partial x} \Phi_{0} \\
& \quad-k_{y}^{2}\left(\frac{\omega_{P e}^{2}}{\omega_{H e}^{2}}+1-\frac{\omega_{P i}^{2}}{\omega^{2}}\right) \Phi_{0}
\end{aligned}
$$

$$
+\frac{\omega_{P e}^{2}}{\omega^{2}}\left(k_{z}^{2}-\kappa_{n} k_{y} \frac{\omega}{\omega_{H e}}\right) \Phi_{0}=0 .
$$

Here $\rho_{e}$ is the Larmour radius of electrons $\rho_{e}=V_{T e} / \omega_{H e}, V_{T e}$ is the thermal velocity of electrons, the plasma frequency of ions is $\omega_{P i}=\left(4 \pi e^{2} N_{0} / M\right)^{1 / 2}, \kappa_{n}$ characterizes the inhomogeneity along the $x$-axis $\kappa_{n}=\frac{\partial N_{0}}{N_{0} \partial x}$, and the LHR frequency is

$\omega_{\mathrm{LHR}}=\frac{\omega_{P i} \omega_{H e}}{\left(\omega_{P e}^{2}+\omega_{H e}^{2}\right)^{1 / 2}}$.

Note, that the LHR frequency varies with plasma concentration, because plasma frequencies of electron and ion depend on the concentration of charged particles. In the geometric optics approximation when the dependence on the $x$-coordinate takes the form

$\Phi_{0} \propto \exp \left(-i \int^{x} k_{x}\left(x_{1}\right) d x_{1}\right)$

we arrive at the following dispersion relation $\omega=\omega(\mathbf{k})$ that follows from Eq. (6)

$$
\begin{gathered}
\rho_{e}^{2}\left(k_{x}^{2}+k_{y}^{2}\right)^{2}+\left(k_{x}^{2}+k_{y}^{2}\right)\left(1-\frac{\omega^{2}}{\omega_{\mathrm{LHR}}^{2}}\right) \\
+\frac{M}{m}\left(k_{z}^{2}-\kappa_{n} k_{y} \frac{\omega}{\omega_{H e}}\right)=0 .
\end{gathered}
$$

Eq. (8) without the last term $\left(\propto \kappa_{n} k_{y}\right)$ presents the well-known dependence of the LH wave frequency on the transverse $k_{x}$ and $k_{y}$ and the longitudinal $k_{z}$ wave numbers taking into account thermal corrections, see, e.g., [9]. The last term in Eq. (8) corresponds to the drift motion of magnetized electrons in the inhomogeneous plasma.

Eq. (6) contains small parameter $\rho_{e}^{2}$ before the highest order derivative. Due to this Eq. (6) has two types of solutions. One of them describes small-scale oscillations for which thermal corrections are definitely important. The second solution corresponds to perturbations with larger scales. In some cases a link between two types of solutions appears in the vicinity of a particular point $x_{0}$. There is some similarity between Eq. (6) that describes LH waves with thermal corrections and equation for UH waves discussed in [10]. The latter is also a differential equation of the fourth order that contains small parameter $\left(r_{d}^{2}\right.$ is the square of the Debye radius) before the highest order derivative. It is known that such equation also has two types of solutions [11]. The small-scale solution corresponds to UH waves that can be trapped inside striations, while the large-scale one is known as the slow extraordinary mode leaking from striations (the so-called Z-mode).

In the next sections we shall discuss the situations when Eqs. (6), (8) describe trapped perturbations with the frequencies either above or below the LHR frequency of the ambient plasma.

\section{Trapping of LH waves in striations}

LH waves excited in heating experiments are expected to have frequencies above the LHR frequency of the ambient plasma. Indeed, LH waves are supposed to be involved in the 
formation of the downshifted maximum (DM) of the secondary electromagnetic radiation. DM is a rather broad structure with the frequency offshift from the heating frequency equal to the LHR frequency [7]. To discuss the trapping of LH oscillations, two-dimensional case $\left(k_{y}=0\right)$ will be considered in this section. Note, that the theory presented in [4-6] does not provide wave trapping in such case. In 2D case Eq. (6) reduces to

$$
\begin{aligned}
\rho_{e}^{2} & \frac{\partial^{3}}{\partial x^{3}} \frac{\omega_{P i}^{2}}{\omega^{2}} \frac{\partial}{\partial x} \Phi_{0}+\frac{\partial}{\partial x}\left(\frac{\omega_{P e}^{2}}{\omega_{H e}^{2}}+1-\frac{\omega_{P i}^{2}}{\omega^{2}}\right) \frac{\partial}{\partial x} \Phi_{0} \\
& +\frac{\omega_{P e}^{2}}{\omega^{2}} k_{z}^{2} \Phi_{0}=0 .
\end{aligned}
$$

It is convenient to present this equation in the following form

$$
\begin{aligned}
\rho_{e}^{2} & \frac{1}{N_{a}} \frac{\partial^{3}}{\partial x^{3}} N_{a} \frac{\partial \Phi_{0}}{\partial x}+\frac{1}{N_{a}} \frac{\partial}{\partial x}\left[N_{a}\left(\frac{\omega^{2}}{\omega_{\mathrm{LHR}}^{2}}-1\right)\right] \frac{\partial \Phi_{0}}{\partial x} \\
& +\frac{M}{m} k_{z}^{2} \Phi_{0}=0 .
\end{aligned}
$$

Here $N_{a}$ is the ambient plasma concentration which consists of the background concentration $N_{0}$ and small negative part $\delta n(x)$ describing the distribution of plasma in a given striation

$N_{a}=N_{0}+\delta n(x)$.

Eq. (10) can be simplified further neglecting the terms $\propto \kappa_{n}$. Indeed for small-scale oscillations when thermal corrections come into play such terms are insignificant. For large-scale oscillations thermal term with the highest order derivative can be neglected. As a result we come to the second-order differential equation in which the mentioned terms can be excluded by standard substitution $\Phi_{0}=\Phi_{1} / \sqrt{n_{r}}$, where $n_{r}$ is the relative plasma density $n_{r}=N_{a} / N_{0}$. The additional terms that appear due to such substitution are insignificant. So, from now on, we deal with the following equation

$\rho_{e}^{2} \frac{\partial^{4} \Phi_{0}}{\partial x^{4}}+\frac{\partial}{\partial x}\left(\frac{\omega^{2}}{\omega_{\text {LHR }}^{2}}-1\right) \frac{\partial \Phi_{0}}{\partial x}+\frac{M}{m} k_{z}^{2} \Phi_{0}=0$.

It should be mentioned that differential equations of the fourth order with small parameter before the highest order derivative are rather common in plasma physics. For example, similar equations have been investigated in relation to the problems of waves transformation in the inhomogeneous plasma (Alfvenic and flute oscillations) [12].

In the geometric optics approximation Eq. (12) reduces to

$\rho_{e}^{2} k_{x}^{4}-A(x) k_{x}^{2}+B=0$,

where

$A(x)=\frac{\omega^{2}}{\omega_{\mathrm{LHR}}^{2}(x)}-1, \quad B=\frac{M}{m} k_{z}^{2}$.

Solutions of Eq. (13) are the following

$k_{x}^{2}=\frac{A(x)}{2 \rho_{H e}^{2}} \pm \sqrt{\frac{A(x)^{2}}{4 \rho_{H e}^{4}}-\frac{B}{\rho_{e}^{2}}}$.

Assume that the minimum of concentration in a given striation is achieved at $x=0$. Let us consider such LH frequencies $\omega$ and the values of the longitudinal wave numbers $k_{z}$ that, first, the coefficient $A(x)$ is positive everywhere and, second, at some particular point of a striation $x=x_{0}$ the equality $A\left(x_{0}\right)=2 \rho_{e} \sqrt{B}$ takes place. It is useful to introduce the function $F=A(x)-2 \rho_{e} \sqrt{B}$ and to analyze its behavior for different frequencies $\omega$ and the model Gaussian distribution of plasma in a striation

$\delta n(x)=-n_{0} \exp \left(-x^{2} / a^{2}\right)$.

It is clear that for the frequencies $\omega$ sufficiently exceeding the LHR frequency (depending on the $k_{z}$ number) the function $F$ is positive everywhere. For smaller frequencies there is a point $x=x_{0}$ where this function goes through zero. Closer to the center $0<x<x_{0}$ it is positive and it becomes negative at the distances $x>x_{0}$. For even more smaller frequencies the function $F$ is negative everywhere. The range of frequencies $\Delta \omega$ within which the function $F$ changes its sign can be estimated as $\Delta \omega \approx \omega_{\text {LHR }}\left(N_{0}\right) \frac{n_{0}}{2 N_{0}}$. This is the frequency range for which the trapping of LH waves exists (as will be shown below).

In order to demonstrate that the trapping indeed exists, the behavior of the group velocity along the $x$-axis should be investigated. The following relation between the group $V_{g}$ and the phase $V_{\text {ph }}$ velocities of LH waves along the $x$-axis can be established with the help of Eq. (13)

$\frac{V_{g}}{V_{\mathrm{ph}}}=\frac{\omega_{\mathrm{LHR}}^{2}}{\omega^{2}}\left(\rho_{e}^{2} k_{x}^{2}-\frac{B}{k_{x}^{2}}\right)$.

According to Eq. (16) the group velocity becomes equal to zero if $\left|k_{x}\right|=k_{x}^{*}$, where

$k_{x}^{* 2}=\sqrt{\frac{M}{m}} \frac{\left|k_{z}\right|}{\rho_{e}}$.

If the wave number of perturbation is large enough $k_{x}^{2}>k_{x}^{* 2}$ the group and the phase velocities have the same signs. For small wave numbers $k_{x}^{2}<k_{x}^{* 2}$ the group and the phase velocities have opposite signs. Note, that according to Eq. (14) the square root becomes equal to zero exactly for $k_{x}=k_{x}^{*}$. It means that inside of a striation at $0<x<x_{0}$ for one (small-scale) solution that corresponds to the sign + before the root in Eq. (14) the group and the phase velocities have the same directions. At the same time for large-scale solution (the sign minus before the root) the group and the phase velocities have opposite directions.

In the inner part of a striation $0<x<x_{0}$ two solutions (large-scale and small-scale ones) are independent. But at the point $x=x_{0}$ two wave numbers become equal to each other. It means that in the vicinity of this point the transformation of waves takes place. To understand the behavior of waves near $x=x_{0}$ more detailed analysis is required. Let us return to our basic differential equation (11). The solution of this equation near $x=x_{0}$ can be obtained in the form of

$\Phi_{0}=f(x) \exp \left(i \frac{\sqrt{A\left(x_{0}\right)}}{\sqrt{2} \rho_{e}} x\right)$,

where $f(x)$ is a slowly varying function to be determined. After the substitution of (18) into Eq. (11) we arrive at the following approximate equation

$2 A\left(x_{0}\right) \frac{\partial^{2} f}{\partial x^{2}}+\left(\frac{A^{2}(x)}{4 \rho_{e}^{2}}-B\right) f=0$. 
In the vicinity of $x=x_{0}$ taking into account that $A(x)$ is a decreasing function of the coordinate $x$, we may set

$A^{2}(x)=4 \rho_{e}^{2} B-\frac{x-x_{0}}{L_{x}}$,

where $L_{x}$ is the characteristic scale of inhomogeneity along the $x$-axis. After the substitution of (20) into Eq. (19) we obtain an equation

$\frac{d^{2} f}{d \xi^{2}}-\xi f=0, \quad$ where $\xi=\frac{x-x_{0}}{\left(8 \rho_{e}^{2} L_{x} A\left(x_{0}\right)\right)^{1 / 3}}$.

Eq. (21) has a well-known solution in the form of the Airy function. For $x<x_{0}$ there are two waves propagating in opposite directions with equal amplitudes. At the same time the chosen solution of Eq. (21) decreases exponentially for $x>x_{0}$. It means that the wave propagating in the region $x \leqslant x_{0}$ towards $x_{0}$ is reflected at this point. The requirement that the function $f(x)$ varies more slowly than the exponent (see Eq. (18)) means

$L_{x} \gg \frac{\rho_{e}}{\sqrt{2} A^{5 / 2}\left(x_{0}\right)}$.

Taking into account Eqs. (18) and (21) we find that for $x \leqslant x_{0}$ the potential $\Phi_{0}$ describes two waves with equal signs of their phases velocities. For brevity we shall call these two waves the small-scale (the sigh + before the root in (14)) and the largescale (the sign - before the root in (14)). It is easy to verify that the phase velocities of these two waves have the same direction (in the positive direction of the $x$-axis). At the same time from Eq. (16) it follows that the directions of the group velocities of the discussed waves are opposite to each other. So, the smallscale wave propagating in the region $x \leqslant x_{0}$ towards $x=x_{0}$ is reflected at this point with the total transformation into the large-scale wave. The latter propagates to the other reflection point in a striation where $A^{2}(x)=4 \rho_{e}^{2} B$. In a symmetric striation this point is $x=-x_{0}$. As a result the trapping of such LH wave takes place. Hence, each given $k_{z}$ number determines a restricted range of frequencies for which the trapping takes place. But the reflection is only a necessary condition for the existence of a stationary state. The appropriate phase relations are discussed in the next section.

\section{Stationary states of the trapped LH waves in striations}

Let us designate by $k_{x}^{(+)}$the wave number of the small-scale wave and by $k_{x}^{(-)}$the wave number of the large-scale wave. The stationary state of the LH wave trapped in a striation exists if two phase integrals between turning points $x_{0}^{(1)}$ and $x_{0}^{(2)}$ obey the following relation

$$
\int_{x_{0}^{(1)}}^{x_{0}^{(2)}}\left(k_{x}^{(+)}\left(x_{1}\right)-k_{x}^{(-)}\left(x_{1}\right)\right) d x_{1}=\pi(2 n+1),
$$

where $n$ is an integer. This relation means that in stationary conditions the phase at a given point $x^{*}$ inside a striation and the phase acquired by the wave after propagation to the first turning point, reflection from it, propagation to the second turning point in the form of another wave, once more reflection, and propagation to the point $x^{*}$ deviate by $2 \pi n$ (where $n$ is an integer). Note, that in contrast with the usual case when the sum of two phase integrals between the turning points should be equal to $\pi(2 n+1)$ in our case the difference of such phase integrals is equal to $\pi(2 n+1)$. The reason for this is that the group and the phase velocities of the large-scale wave have opposite directions.

The relation (23) can be fulfilled only for some particular discrete set of frequencies $\omega_{n}$ of the LH wave. To find the set of eigenfrequencies let us suppose that $A \gg \sqrt{A^{2}-4 \rho_{e}^{2} B}$. In such case the wave numbers determined by Eq. (14) are the following

$k_{x}^{( \pm)}=\sqrt{\frac{A}{2 \rho_{H e}^{2}}}\left(1 \pm \frac{\sqrt{A^{2}-4 \rho_{e}^{2} B}}{2 A}\right)$.

It is convenient to present the coefficient $A$ in the form

$A(x)=\frac{\left(\omega_{0}+\delta \omega_{n}\right)^{2}}{\omega_{H i} \omega_{H e}}\left(1+\frac{\omega_{H i} \omega_{H e}}{\omega_{P i}^{2}\left(N_{0}\right)}-\frac{\delta n(x)}{N_{a}} \frac{\omega_{H i} \omega_{H e}}{\omega_{P i}^{2}\left(N_{0}\right)}\right)-1$.

Here $\omega_{0}$ is an averaged LH frequency determined by

$\omega_{0}=\omega_{\mathrm{LHR}}\left(N_{0}\right)\left(1+2 \rho_{e} \sqrt{\frac{M}{m}}\left|k_{z}\right|\right)^{1 / 2}$

and $\delta \omega_{n}$ are small corrections associated with discrete eigenstates. Note that the frequency $\omega_{0}$ is larger than the LHR frequency of the ambient plasma due to thermal corrections and finite longitudinal wave number $k_{z}$.

With the help of Eqs. (25)-(26) the relation (23) can be presented as follows

$\int_{x_{0}^{(1)}}^{x_{0}^{(2)}} \sqrt{\frac{2 \omega_{0} \delta \omega_{n}}{\omega_{\mathrm{LHR}}^{2}\left(N_{0}\right)}-\frac{\omega_{0}^{2}}{\omega_{P i}^{2}} \frac{\delta n(x)}{N_{0}}} d x=\pi(2 n+1) \rho_{e}$.

After the substitution of the model plasma distribution in a striation (15) we arrive at the following equation for eigenfrequencies $\delta \omega_{n}$

$$
\begin{aligned}
& \frac{\omega_{0}}{\omega_{P i}} \int_{x_{0}^{(1)}}^{x_{0}^{(2)}} \sqrt{\frac{n_{0}}{N_{0}} \exp \left(-\frac{x^{2}}{a^{2}}\right)+2 \frac{\delta \omega_{n}}{\omega_{0}} \frac{\omega_{P e}^{2}}{\omega_{H e}^{2}}\left(1+\frac{\omega_{H e}^{2}}{\omega_{P e}^{2}\left(N_{0}\right)}\right)} d x \\
& =\pi(2 n+1) \rho_{e} .
\end{aligned}
$$

Not far from the center of a striation we may use the approximation $\exp \left(-x^{2} / a^{2}\right) \approx 1-x^{2} / a^{2}$. As a result we rewrite Eq. (28) in the following form

$$
\begin{aligned}
& \frac{\omega_{0}}{\omega_{P i}} \int_{x_{0}^{(1)}}^{x_{0}^{(2)}} \sqrt{\frac{n_{0}}{N_{0}}+2 \frac{\delta \omega_{n}}{\omega_{0}} \frac{\omega_{P e}^{2}}{\omega_{H e}^{2}}\left(1+\frac{\omega_{H e}^{2}}{\omega_{P e}^{2}\left(N_{0}\right)}\right)-\frac{n_{0}}{N_{0}} \frac{x^{2}}{a^{2}}} d x \\
& =\pi(2 n+1) \rho_{e} .
\end{aligned}
$$


It is convenient to introduce a new parameter $\epsilon_{n}$ and a new variable $\xi$ instead of $x$

$\epsilon_{n}=1+2 \frac{N_{0}}{n_{0}} \frac{\delta \omega_{n}}{\omega_{0}} \frac{\omega_{P e}^{2}}{\omega_{H e}^{2}}\left(1+\frac{\omega_{H e}^{2}}{\omega_{P e}^{2}\left(N_{0}\right)}\right), \quad \xi=\frac{x}{a}$.

After the integration with respect to $x$ in Eq. (29) we come to the relation

$\frac{\omega_{0}}{\omega_{P i}} \sqrt{\frac{n_{0}}{N_{0}}} \epsilon_{n}=2(2 n+1) \frac{\rho_{e}}{a}$.

This relation determines the set of discrete eigenfrequencies as the functions of parameters of a striation (its relative depth $\frac{n_{0}}{N_{0}}$ and its halfwidth $a$ ). It is clear from Eq. (28) that the turning points for $x \leqslant a$ appear only if the eigenfrequencies $\delta \omega_{n} / \omega_{0}$ are negative. There is a finite set of such frequencies

$\frac{\delta \omega_{n}}{\omega_{0}} \approx-\left(1-2 \sqrt{\frac{N_{0}}{n_{0}}} \frac{\omega_{P i}}{\omega_{0}} \frac{\rho_{e}}{a}(2 n+1)\right) \frac{n_{0}}{2 N_{0}} \frac{\omega_{e}^{2}}{\omega_{P e}^{2}}$.

It is seen that the broader and the deeper the striation is, the more eigenfrequencies corresponding to the trapped LH waves exist in it. Taking into account the value of the averaged frequency $\omega_{0}$, we find the eigenfrequencies $\omega_{n}=\omega_{0}+\delta \omega_{n}$ in the form

$$
\begin{aligned}
\omega_{n} \approx & \omega_{\mathrm{LHR}}\left(N_{0}\right)\left[1+\sqrt{\frac{M}{m}} \rho_{e}\left|k_{z}\right|\right. \\
& \left.-\left(1-2 \sqrt{\frac{N_{0}}{n_{0}}} \frac{\omega_{P i}}{\omega_{0}} \frac{\rho_{e}}{a}(2 n+1)\right) \frac{n_{0}}{2 N_{0}} \frac{\omega_{H e}^{2}}{\omega_{P e}^{2}}\right] .
\end{aligned}
$$

It is clearly seen from Eq. (33) that due to the longitudinal correction (if the factor $\sqrt{\frac{M}{m}} \rho_{e}\left|k_{z}\right|$ is not too small) some of the eigenfrequencies are definitely higher than the LHR frequency of the background plasma $\omega_{\mathrm{LHR}}\left(N_{0}\right)$. At the same time there are eigenstates with the frequencies slightly less than $\omega_{\text {LHR }}\left(N_{0}\right)$ but still higher than the minimal LHR frequency in the center of a striation. Estimates based on Eq. (33) show that for striation with $10 \%$ depletion and a half-width $a=5 \mathrm{~m}$, the amount of trapped LH modes comprises $n_{\max } \sim 10$ for a given $k_{z}$ number. It can be verified that the $k_{x}$ numbers of the trapped modes are rather large, that is the oscillations are small-scale ones. Indeed, it follows from Eqs. (24), (26) that for typical HF range $k_{z}$ numbers $k_{z} \sim(2-4) \times 10^{-4} \mathrm{~cm}^{-1}$ the characteristic sizes for $\mathrm{LH}$ oscillations along the $x$-axis are of the order of $l_{x} \sim 5-10 \mathrm{~cm}$. It is approximately one order of magnitude larger than the Debye size. For natural plasma depletions in the upper ionosphere with the relative depth $n_{0} / N_{0} \approx(5-10) \%$ and the halfwidth $a=25 \mathrm{~m}$ an estimate of the amount of LH modes trapped in a plasma cavity gives the value $n_{\max } \approx 6-8$ (depending on the magnitude of the plasma frequency of ions).

The same results can be obtained if we seek for the solution in the form (18) with the minus sign in the exponent

$$
\Phi_{0}=f(x) \exp \left(-i \frac{\sqrt{A\left(x_{0}\right)}}{\sqrt{2} \rho_{e}} x\right)
$$

and in Eq. (24) we also use the minus sign before the square root

$k_{x}^{( \pm)}=-\sqrt{\frac{A}{2 \rho_{e}^{2}}}\left(1 \pm \frac{\sqrt{A^{2}-4 \rho_{H e}^{2} B}}{2 A}\right)$.

So we have found solutions that correspond to the trapped $\mathrm{LH}$ modes inside striations with the set of eigenfrequencies (33).

\section{Discussion and conclusion}

We have investigated conditions for trapping of LH waves in artificial plasma depletions (striations) created in heating experiments. It is shown that there is a restricted range of frequencies below and above the LHR frequency of the ambient plasma within which the trapping can take place. This range of frequencies depends on the longitudinal wave number $k_{z}$ of the excited waves and the relative depletion of plasma in striations. The increase of $k_{z}$ number provides the trapping of LH oscillations with higher frequencies compared to the LHR frequency of the background plasma. In heating experiments the magnitude of the longitudinal wave number for the excited LH waves is determined by the matching conditions between the corresponding wave numbers of the interacting $\mathrm{UH}$ and $\mathrm{LH}$ waves. For typical wave numbers $k_{z} \sim\left(4 \times 10^{-4}-10^{-3}\right) \mathrm{cm}^{-1}$ the excited LH waves trapped in striations can have frequencies exceeding the LHR frequency up to $\sim(10-25) \%$. As it was mentioned before, trapped LH waves should have higher intensities than those propagating freely from striations. This in turn should influence the magnitude of the downshifted maximum in the secondary stimulated emissions.

The obtained above results can be helpful in explanation of LH waves trapping in natural plasma cavities in the Earth's upper ionosphere. The Freja satellite detected shallow $n_{0} / N_{0} \sim$ $0.01-0.1$ and rather broad $a \sim 20-100 \mathrm{~m}$ plasma depletions in the upper ionosphere associated with the LH waves "spikelets" $[2,3]$. In such depletions the drift of magnetized electrons is too weak to provide the trapping by the mechanism discussed in [4-6]. At the same time our approach can easily explain the trapping of LH waves in such shallow cavities. Indeed, in the upper ionosphere the Larmour frequency of electrons $\omega_{H e}$ becomes of the order or even larger than the plasma frequency $\omega_{P e}$. Due to this even in shallow depletions significant amount of localized eigenstates with the frequencies around the LHR frequency can exist.

\section{Acknowledgements}

This work was supported by the Leverhulme Trust Grant Ref. Number F00185R.

\section{References}

[1] J.L. Vago, et al., J. Geophys. Res. 97 (1992) 16935.

[2] P.O. Dovner, et al., Geophys. Res. Lett. 21 (1994) 1827.

[3] H.L. Pećsely, et al., J. Geophys. Res. 101 (1996) 5299.

[4] P.W. Schuck, et al., J. Geophys. Res. 103 (1998) 6935.

[5] P.W. Schuck, et al., IEEE Trans. Plasma Sci. 31 (2003) 1125. 
[6] P.W. Schuck, et al., J. Geophys. Res. 109 (2004) A01310.

[7] T. Leyser, Geophys. Res. Lett. 18 (1991) 408.

[8] A.V. Gurevich, et al., Phys. Lett. A 231 (1997) 97.

[9] V.D. Shapiro, et al., Phys. Fluids B 5 (1993) 3148.
[10] N.D. Borisov, T.R. Robinson, Phys. Lett. A 315 (2003) 126.

[11] E. Mjølhus, J. Plasma Phys. 29 (1983) 195.

[12] A.V. Timofeev, in: Reviews of Modern Physics, vol. 17, Consultants Bureau, NY, 1992, p. 193. 